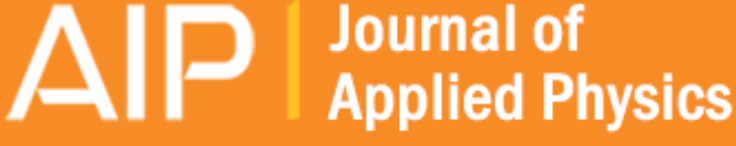

\section{Simulation and analysis of grating-integrated quantum dot infrared detectors for spectral response control and performance enhancement}

Jun Oh Kim, Zahyun Ku, Sanjay Krishna, Sang-Woo Kang, Sang Jun Lee, Young Chul Jun, and Augustine Urbas

Citation: Journal of Applied Physics 115, 163101 (2014); doi: 10.1063/1.4871855

View online: http://dx.doi.org/10.1063/1.4871855

View Table of Contents: http://scitation.aip.org/content/aip/journal/jap/115/16?ver=pdfcov

Published by the AIP Publishing

\section{Articles you may be interested in}

Simulating the spectral response of quantum dot-in-well infrared photodetectors from eight band k.p method J. Appl. Phys. 116, 203701 (2014); 10.1063/1.4902385

Enhanced normal incidence photocurrent in quantum dot infrared photodetectors

J. Vac. Sci. Technol. B 29, 03 C123 (2011); 10.1116/1.3562186

Enhanced optical response in doubly waveguided plasmonic gratings

Appl. Phys. Lett. 93, 093113 (2008); 10.1063/1.2978236

Origin of detection wavelength tuning in quantum dots-in-a-well infrared photodetectors

Appl. Phys. Lett. 88, 251107 (2006); 10.1063/1.2216920

High-speed $>90 \%$ quantum-efficiency $p-i-n$ photodiodes with a resonance wavelength adjustable in the $795-835$ nm range

Appl. Phys. Lett. 74, 1072 (1999); 10.1063/1.123485

\section{The new SR865 2 MHz Lock-In Amplifier ... \$7950}

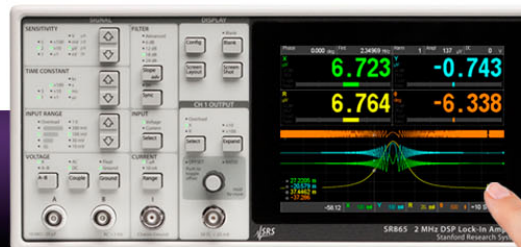

NRS Stanford Research Systems

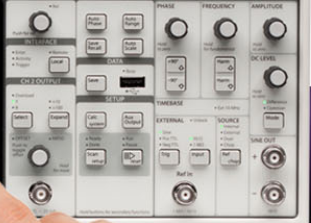

(๑)
Intuitive front-panel operation

Touchscreen data display

Save data \& screen shots to USB flash drive

Embedded web server and iOS app

. Synch multiple SR865s via $10 \mathrm{MHz}$ timebase I/O

View results on a TV or monitor (HDMl output)

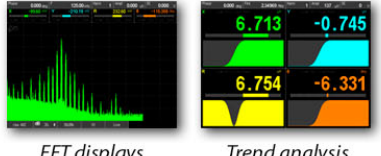

FFT displays
$.1 \mathrm{mHz}$ to $2 \mathrm{MHz}$

$\cdot 2.5 \mathrm{nV} / \sqrt{ } \mathrm{Hz}$ input noise

. $1 \mu$ s to 30 ks time constants

- 1.25 MHz data streaming rate

Sine out with DC offset

GPIB, RS-232, Ethernet \& USB 


\title{
Simulation and analysis of grating-integrated quantum dot infrared detectors for spectral response control and performance enhancement
}

\author{
Jun Oh Kim, ${ }^{1,3, a)}$ Zahyun Ku, ${ }^{2, a)}$ Sanjay Krishna, ${ }^{1}$ Sang-Woo Kang, ${ }^{3}$ Sang Jun Lee, ${ }^{3}$ \\ Young Chul Jun, ${ }^{4, b)}$ and Augustine Urbas ${ }^{2, b)}$ \\ ${ }^{1}$ Center for High Technology Materials, University of New Mexico, Albuquerque, New Mexico 87106, USA \\ ${ }^{2}$ Air Force Research Laboratory, Wright-Patterson Air Force Base, Ohio 45433, USA \\ ${ }^{3}$ Division of Industrial Metrology, Korea Research Institute of Standards and Science, Daejeon 305-340, \\ South Korea \\ ${ }^{4}$ Department of Physics, Inha University, Incheon 402-751, South Korea
}

(Received 8 January 2014; accepted 8 April 2014; published online 22 April 2014)

\begin{abstract}
We propose and analyze a novel detector structure for pixel-level multispectral infrared imaging. More specifically, we investigate the device performance of a grating-integrated quantum dots-in-a-well photodetector under backside illumination. Our design uses 1-dimensional grating patterns fabricated directly on a semiconductor contact layer and, thus, adds a minimal amount of additional effort to conventional detector fabrication flows. We show that we can gain wide-range control of spectral response as well as large overall detection enhancement by adjusting grating parameters. For small grating periods, the spectral responsivity gradually changes with parameters. We explain this spectral tuning using the Fabry-Perot resonance and effective medium theory. For larger grating periods, the responsivity spectra get complicated due to increased diffraction into the active region, but we find that we can obtain large enhancement of the overall detector performance. In our design, the spectral tuning range can be larger than $1 \mu \mathrm{m}$, and, compared to the unpatterned detector, the detection enhancement can be greater than $92 \%$ and $148 \%$ for parallel and perpendicular polarizations. Our work can pave the way for practical, easy-to-fabricate detectors, which are highly useful for many infrared imaging applications. (C) 2014 AIP Publishing LLC. [http://dx.doi.org/10.1063/1.4871855]
\end{abstract}

\section{INTRODUCTION}

Infrared (IR) detectors and imagers working in the 3-12 $\mu \mathrm{m}$ range can provide information about the temperature and chemical composition of a target object, and have various applications in thermal imaging and medical diagnostics. ${ }^{1-7}$ IR focal plane arrays are IR imaging cameras consisting of two dimensional arrays of such IR photodetectors placed at the focal plane of imaging systems. They have been widely used for night vision, military surveillance, environmental monitoring, etc. Multispectral detectors, which can provide spectral resolution and enable multiband detection, are highly beneficial for these applications and are being actively studied. ${ }^{8-13}$ Usually, a broadband IR camera is used to obtain the spatial distribution of IR radiation (i.e., IR images), together with dispersive elements (such as spinning filter wheels or prisms), to get the spectral information. However, these mechanical parts increase the cost and complexity of IR cameras significantly.

Recently, metallic gratings or plasmonic structures have been integrated with IR photodetectors at the pixel level to gain spectral selectivity. ${ }^{14-21}$ In addition, the use of such structures can improve the detector efficiency by the many passes light makes or by the increased field intensity in a detector cell. This pixel-level structure can be combined

\footnotetext{
a) J. O. Kim and Z. Ku contributed equally to this work.

b)Electronic addresses: youngchul.jun@inha.ac.kr and Augustine.Urbas@ wpafb.af.mil
}

with efficient compressive sensing algorithms to reduce the data volume while increasing the information from the detector. However, such perforated metal structures can complicate the device fabrication and suffer from additional optical losses in the metal layer.

Here, we propose and analyze an alternative, easy-tofabricate photodetector structure for pixel-level multispectral IR imaging. We investigate 1-dimensional (1D) grating structures patterned directly on a semiconductor contact layer in IR detectors. More specifically, we theoretically analyze the device performance of a quantum dots-in-a-well (DWELL) photodetector integrated with a 1D surface grating. DWELL structures have been the focus of significant interest recently, ${ }^{22-25}$ because they combine the superior peak wavelength control of quantum well (QW) IR detectors with the reduced dark current and normal incidence operation of quantum dot (QD) IR detectors. We show that our simple grating structure can have wide-range control of spectral response as well as large overall detection enhancement, depending on the choice of grating parameters. This can be achieved with a minimum level of additional effort for device fabrication. We find that, for small grating periods $(\sim 1 \mu \mathrm{m})$, the spectral responsivity gradually changes with grating parameters. But, for larger grating periods, the responsivity spectra get more complicated due to increased diffraction into the substrate. In this case, gradual tuning of the spectral response becomes more difficult, but we find that we can obtain large enhancement of the overall detector performance instead. In our analysis, we use the anisotropic 
dielectric constants for the active DWELL layer in order to account for the different absorption efficiencies of QDs into different directions. This is also helpful for understanding the physical origin of the spectral response in our device. We use the Fabry-Perot (FP) resonance and effective medium theory to understand the spectral response changes and the behavior of subwavelength gratings. In fact, this also highlights the uniqueness of $\mathrm{QD}$ detectors compared to $\mathrm{QW}$ detectors. Grating structures have been used to enable optical coupling into QW IR detectors, ${ }^{26,27}$ because they have light absorption only in one direction (along the growth direction). However, here we use subwavelength gratings patterned on a semiconductor contact to adjust resonance and light absorption conditions in the active QD layer. Considering anisotropic absorption efficiencies of QDs into different directions, we show that our grating-integrated DWELL detectors can enable wide-range control of spectral response as well as large overall detection enhancement.

\section{DEVICE STRUCTURE AND ANALYSIS METHOD}

Figure 1 shows a schematic of the IR photodetector structure that we studied (note that only one unit cell is shown here). Such structures can be made by substrate removal and patterning of a grating structure on the surface of the flipped semiconductor contact layer. ${ }^{17}$ We assume 20 stacks of InAs QDs embedded in $3.5 \mathrm{~nm}$ thick $\mathrm{In}_{0.15} \mathrm{Ga}_{0.85} \mathrm{As} / \mathrm{Al}_{0.08} \mathrm{Ga}_{0.92} \mathrm{As}$ QWs, each separated by a $50 \mathrm{~nm}$ thick $\mathrm{Al}_{0.08} \mathrm{Ga}_{0.92} \mathrm{As}$ barrier. In our simulation, we model this active layer as an $1120 \mathrm{~nm}$ thick effective absorber whose anisotropic dielectric constants are taken from the literature. ${ }^{28,29} \mathrm{We}$ use the anisotropic dielectric constants to account for the different absorption efficiencies of QDs into $x$ (or $y$ ) and $z$ directions. The QD absorption covers the mid-IR range of $4-8 \mu \mathrm{m}$. The active absorber is sided by a $1500 \mathrm{~nm}$ thick top contact layer and a $200 \mathrm{~nm}$ thick bottom contact layer. Both contact layers are made of $\mathrm{n}$-GaAs $\left(\mathrm{N}_{\mathrm{D}}=2 \times 10^{18} \mathrm{~cm}^{-3}\right)$. We also assume the

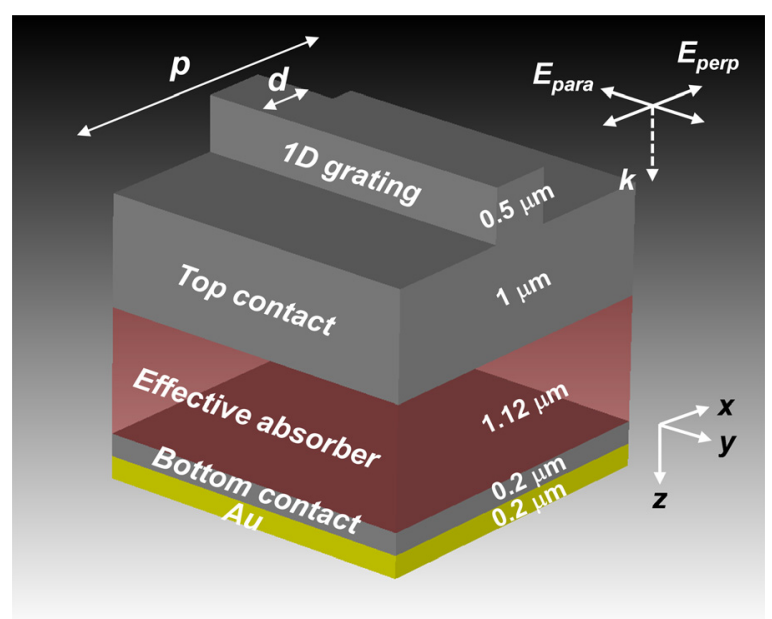

FIG. 1. Schematic of a grating-integrated DWELL IR detector. The DWELL active area is labeled as "Effective absorber." Both top and bottom $\mathrm{n}$-GaAs contact layers (dark gray regions) have a doping density $\mathrm{N}_{\mathrm{D}}=2 \times 10^{18} \mathrm{~cm}^{-3}$. The top semiconductor contact layer is patterned to be a 1-dimensional grating. The grating period $p$ and pattern width $d$ are gradually varied to investigate the device performance of a grating-integrated DWELL photodetector.
$200 \mathrm{~nm}$ thick gold layer is deposited on the bottom n-GaAs layer. We pattern the top contact GaAs layer to be a 1D grating with a period $p$ and a width $d$ (Fig. 1). The grating thickness is fixed at $500 \mathrm{~nm}$. This semiconductor grating is relatively easy to fabricate and compatible with conventional detector fabrication procedures. We gradually vary the grating parameters and numerically investigate the spectral response of the DWELL detector. In addition, we evaluate the overall device performance (ODP) and study detection enhancement in these IR detectors with the overlaid grating.

For this purpose, we perform 3D full field electromagnetic simulations using a finite integration technique (FIT). ${ }^{30}$ The absorption in the QD active layer is calculated for each illumination wavelength. We assume that light is incident on the grating side (i.e., backside illumination) and has a blackbody spectrum of $\mathrm{T}=423 \mathrm{~K}$ (this is the temperature used in Ref. 17). The absorbed power per unit volume can be obtained from the divergence of the Poynting vector ${ }^{31}$

$$
\nabla \cdot \vec{S}=\frac{1}{2} \omega \operatorname{Im}[\varepsilon(\omega)]|E(\omega)|^{2},
$$

where $\omega$ is the angular frequency. The total absorption in the active region can be obtained by integrating this expression over the whole active region

$$
A(\omega)=\sum_{i} A_{i}(\omega)=\sum_{i} \int \frac{1}{2} \omega \operatorname{Im}\left[\varepsilon_{i}(\omega)\right] \cdot\left|E_{i}(\omega)\right|^{2} d V,
$$

where $i$ 's are $x, y$, and $z$. Here, $\left|E_{i}(\omega)\right|^{2}$ is the electric field intensity, and $\varepsilon_{i}(\omega)$ is the anisotropic dielectric constant in the $i$ direction. The calculated absorption spectrum is used to evaluate the spectral responsivity $R(\omega)=A(\omega) \cdot R_{423 K}$, where $R_{423 K}$ can be obtained from a blackbody spectrum at $423 \mathrm{~K}$ and the equivalent spectral current density $J(\omega)=N_{0}(\omega) \cdot e$. Here, $N_{0}(\omega)$ is the incident photon flux, and $e$ is the electron charge. The same numerical technique can be applied to other types of photodetectors working at different wavelengths.

In the simulation, we use the dielectric constants of gold measured using spectral ellipsometry. The dielectric constants of the top and bottom n-GaAs contact layers are obtained from the Drude model with experimental parameters from Ref. 32. The dielectric constants of doped semiconductors vary depending on the doping level and wavelength. In fact, heavily-doped semiconductors can even be metallic $(\operatorname{Re}[\varepsilon(\omega)]<0)$ at IR wavelengths. ${ }^{33}$ But, for a given doping density $\left(\mathrm{N}_{\mathrm{D}}=2 \times 10^{18} \mathrm{~cm}^{-3}\right)$ and the wavelength range considered in our simulation, $\mathrm{n}-\mathrm{GaAs}$ is a dielectric material (i.e., $\operatorname{Re}[\varepsilon(\omega)]$ is well above zero). So, we use a dielectric grating structure for our detector design.

\section{RESULTS AND DISCUSSION}

We gradually vary the groove period $p$ and width $d$ and numerically calculate the spectral response of the DWELL detector for the parallel and perpendicular polarization of light to the groove. Figure 2 shows the obtained spectral responsivity for four grating periods $(p=1-4 \mu \mathrm{m})$. We present the spectra data using the colormap to show the spectral 

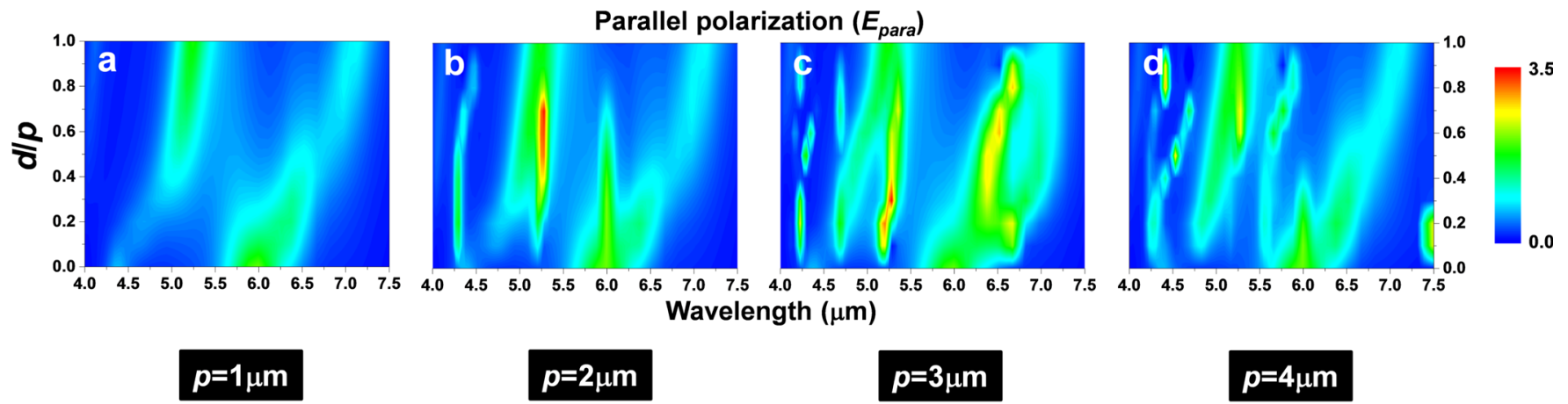

$p=2 \mu \mathrm{m}$

$p=3 \mu m$

$p=4 \mu \mathrm{m}$
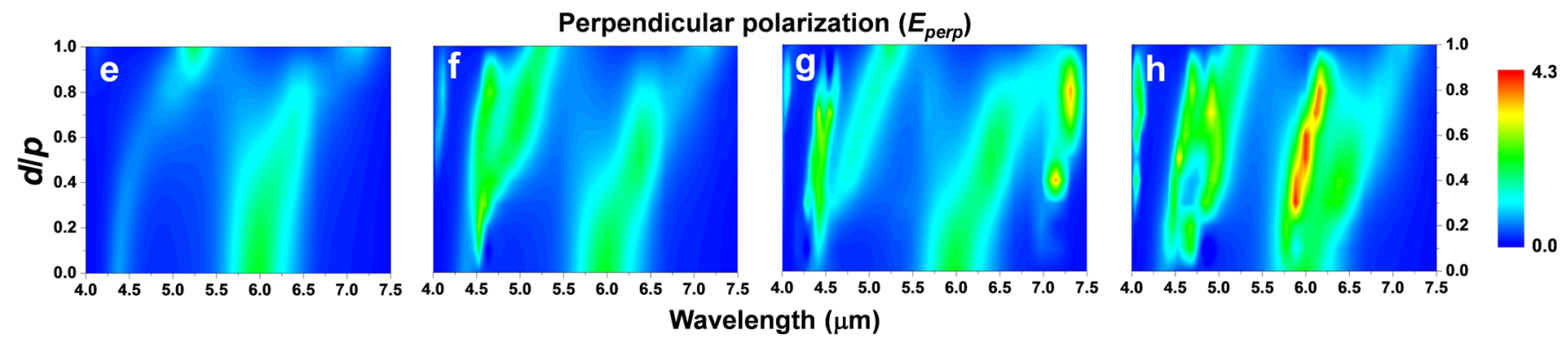

FIG. 2. Spectral responsivity colormaps for parallel (a)-(d) and perpendicular (e)-(h) polarizations. The grating period $p$ is varied from $1 \mu \mathrm{m}$ to $4 \mu \mathrm{m}$. For each period, the responsivity map is presented in color as a function of wavelength and the ratio $d / p$. For a small grating period $(p=1 \mu \mathrm{m})$, the detector response gradually deviates from that of the unpatterned detector as $d$ decreases. For larger periods, the spectral response gets more complicated for both polarizations.

changes clearly. The wavelength and the ratio $d / p$ are varied continuously, and the color changes from blue to red for increased responsivity. Here, two extreme cases for the width $d$ are (i) $d=0 \cdot p$ (i.e., no groove pattern, but the top contact layer is thinned down to $1 \mu \mathrm{m}$ ) and (ii) $d=1 \cdot p$ (i.e., the unpatterned structure with an original $1.5 \mu \mathrm{m}$ thick top contact layer).

We can observe the drastic changes of the responsivity spectra, depending on the grating parameters $(p$ and $d$ ). The upper panel in Fig. 2 shows the spectral responsivity maps for the parallel polarization. For a small periodicity $(p=1 \mu \mathrm{m})$, the responsivity gradually deviates from that of the unpatterned device $(d=1 \cdot p)$ as the width $d$ decreases. Two response peaks at $5.3 \mu \mathrm{m}$ and $7.1 \mu \mathrm{m}(d=1 \cdot p)$ gradually blue-shift as $d$ decreases. Those two peaks move to $4.4 \mu \mathrm{m}$ and $6 \mu \mathrm{m}$, respectively, at $d=0 \cdot p$. This spectral shift occurs gradually as the grating width changes. Therefore, we can precisely tune the detector response (both response peak position and intensity) by simply patterning a $1 \mathrm{D}$ grating on a semiconductor contact layer. This grating structure can be useful for providing tunable spectral selectivity at the pixel level. For a larger periodicity $(p=2-4 \mu \mathrm{m})$, a number of sharp peaks appear, and the responsivity spectra get more complicated. These spectra can be significantly different from that of unpatterned devices.

We also calculate the responsivity for the perpendicular polarization of light to the groove (Lower panel in Fig. 2). For a small periodicity, two responsivity peaks again blueshift gradually as the width $d$ decreases (but two polarizations still exhibit different behavior, as shown in Figs. 2(a) and 2(e). The perpendicular polarization results in larger responsivity at the longer wavelength peak). For a larger periodicity, a number of sharper and stronger peaks appear, and the responsivity spectra get more complicated again.
The spectral response peaks generally blue-shift, as the pattern width $d$ decreases. We can explain this trend using effective medium theory. Responsivity peaks for unpatterned detectors appear at 4.1, 5.3, and 7.1 $\mu \mathrm{m}$ (for $d=1 \cdot p$; this corresponds to a $1.5 \mu \mathrm{m}$ thick top contact layer), and at 4.4 and $6 \mu \mathrm{m}$ (for $d=0 \cdot p$; this corresponds to a $1 \mu \mathrm{m}$ thick top contact layer). These response peaks can be understood as the FP resonances in an air/dielectric/metal system. From a simple FP model, we find that these responsivity peaks correspond to the FP resonance of order $m=5,4,3$ (for $d=1 \cdot p$ ), and $m=4,3$ (for $d=0 \cdot p$ ). As $d / p$ decreases from 1 to 0 , the responsivity peaks tend to move along a line of the same order FP resonance. According to effective medium theory, ${ }^{34-36}$ a subwavelength grating can be replaced by a homogeneous, isotropic layer with an appropriate effective index. The effective indices of a $1 \mathrm{D}$ subwavelength grating are given by

$$
\left.n_{\text {eff }}^{2}(\omega)\right|_{T E}=f \cdot n_{n+}^{2}(\omega)+(1-f) \cdot n_{\text {air }}^{2},
$$

for the parallel polarization and

$$
\left.n_{\text {eff }}^{-2}(\omega)\right|_{T M}=f \cdot n_{n+}^{-2}(\omega)+(1-f) \cdot n_{\text {air }}^{-2},
$$

for the perpendicular polarization. Here, $f$ is the grating fill factor (i.e., $d / p$ ), and $n_{n+}$ and $n_{\text {air }}$ are the refractive indices of n-GaAs and air. Figures 3(a) and 3(b) show the calculated effective indices for the parallel and perpendicular polarizations, as a function of wavelength and the ratio $d / p$. The effective index for the parallel polarization changes smoothly over the whole region of $d / p$. In contrast, the effective index for the perpendicular polarization does not vary at small $d / p$ 's but increases rapidly at larger $d / p$ 's. This trend agrees with spectral changes in Fig. 2. When these effective 


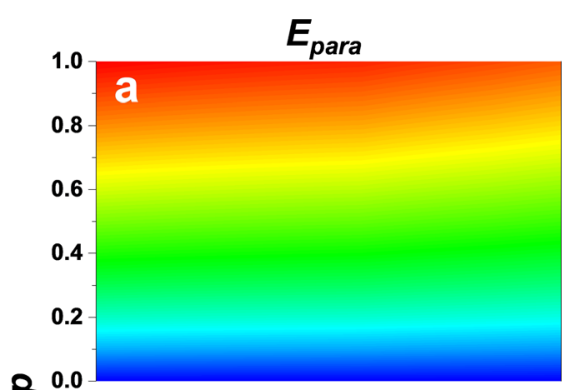

용

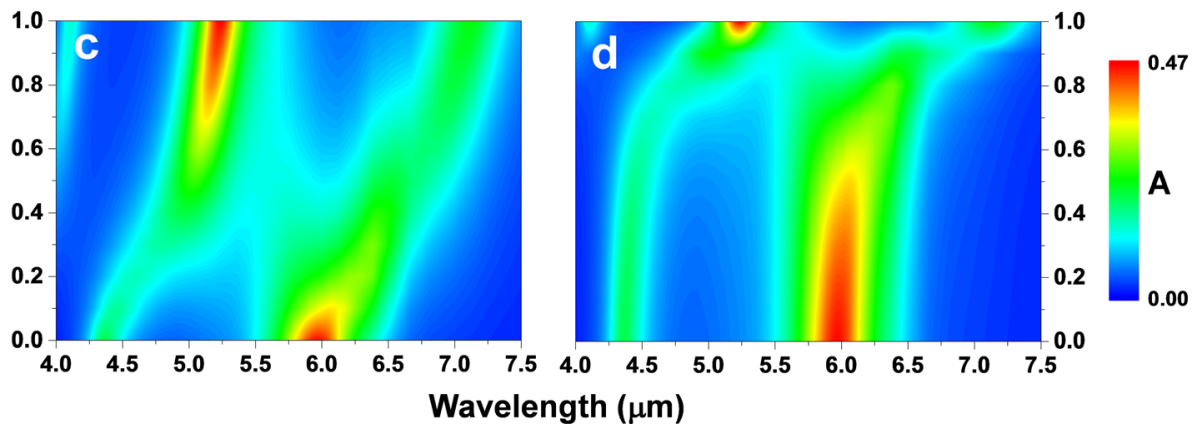

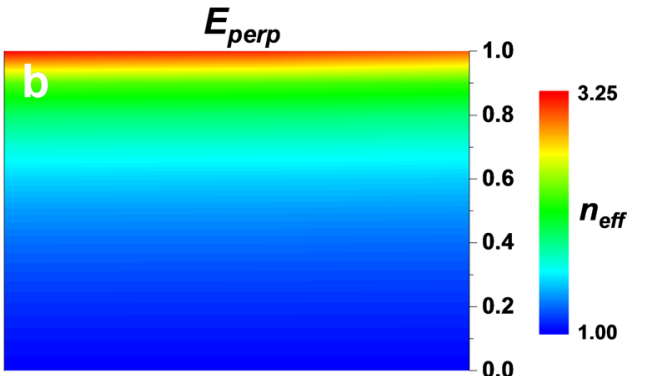

FIG. 3. Effective indices of a 1D grating as a function of wavelength and the ratio $d / p$ for (a) the parallel polarization and (b) the perpendicular polarization. Note that $d=1 \cdot p$ corresponds to a $1.5 \mu \mathrm{m}$ thick top contact layer and $d=0 \cdot p$ corresponds to a $1 \mu \mathrm{m}$ top contact layer. (c) and (d) are the total absorption for the parallel and perpendicular polarizations, which are obtained from a rigorous coupled wave analysis using the effective indices in (a) and (b). Although these are semianalytic calculations, they capture all essential features obtained from the purely numerical method (Figs. 2(a) and 2(e)). indices are plugged into a rigorous coupled wave analysis, we could obtain results (Figs. 3(c) and 3(d)) comparable with those from the FIT based simulations.

For a larger periodicity, a number of sharp peaks appear due to increased diffraction into the active region. Diffracted light with parallel incident polarization is still strongly polarized in the $y$ direction. Thus, the light absorption in the QD active region mainly originates from the $\mathrm{A}_{\mathrm{y}}$ component. On the other hand, perpendicularly polarized light diffracts into both $x$ and $z$ directions, and both $\mathrm{A}_{\mathrm{x}}$ and $\mathrm{A}_{\mathrm{z}}$ components contribute to spectral response. Figure 4 shows this behavior clearly. Figures 4(a)-4(d) are the colormaps of $A_{y}$ for the parallel polarization, while Figs. 4(e)-4(h) are the colormaps of $A_{x}$ and Figs. 4(i)-4(1) are the colormaps of $A_{z}$ for the perpendicular polarization. The absorption spectra get more complicated for a larger periodicity due to the increased
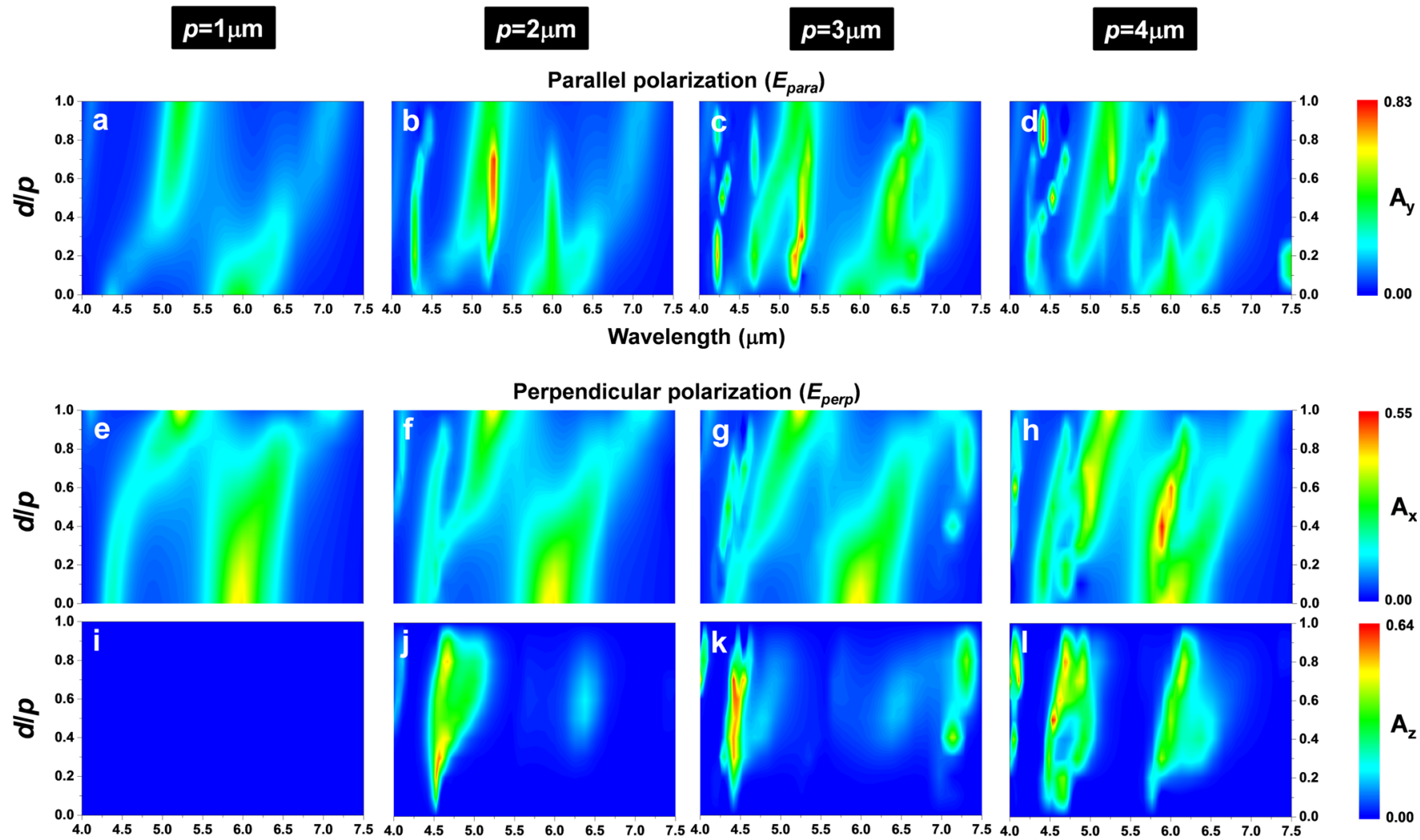

Wavelength $(\mu \mathrm{m})$

FIG. 4. Colormaps of separate absorption $\left(A_{y}, A_{x}\right.$, or $\left.A_{z}\right)$ in the active layer. (a)-(d) are the colormaps of $A_{y}$ for the parallel polarization, (e)-(h) are the colormaps of $A_{x}$ for the perpendicular polarization, and (i)-(1) are the colormaps of $A_{z}$ for the perpendicular polarization. 


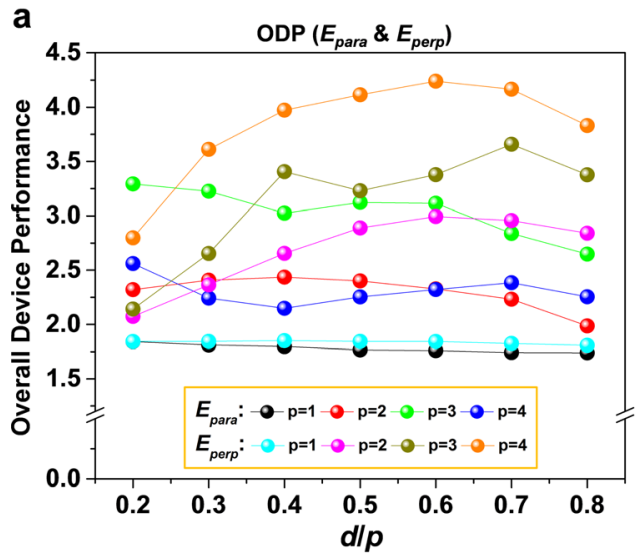

b

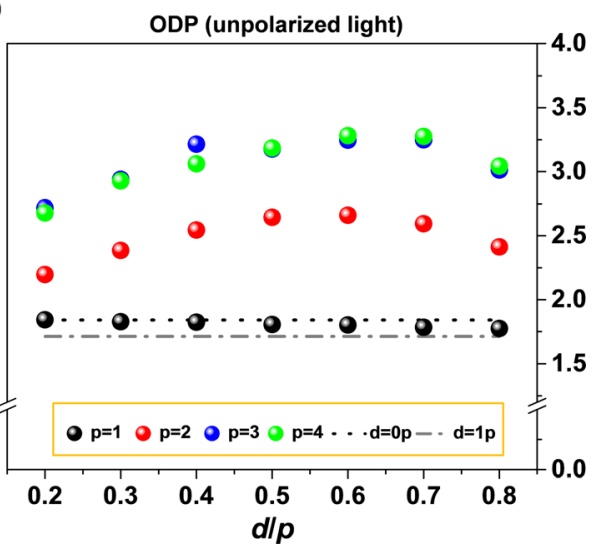

FIG. 5. Overall device performance (ODP). (a) The ODP as a function of the ratio $d / p$ for different grating periods (in different colors). (b) The ODP for unpolarized light (i.e., average of two polarizations). For a small grating period ( $p=1 \mu \mathrm{m})$, the ODP remains almost the same as the unpatterned detector. But for larger grating periods $(p=2-4 \mu \mathrm{m})$, the ODP varies considerably depending on the ratio $d / p$ and can be enhanced significantly. The gray dotted-dashed line is the ODP of the unpatterned detector (ODP 1.71) for a $1.5 \mu \mathrm{m}$ thick top contact $(d=1 \cdot p)$, and the black dotted line is the ODP of the unpatterned detector (ODP 1.84) for a $1 \mu \mathrm{m}$ thick top contact $(d=0 \cdot p)$.

grating diffraction. For example, for the perpendicular polarization, there is essentially no diffraction into the $z$ direction for a small period $(p=1 \mu \mathrm{m})$. However, for larger periods, we have stronger diffraction, and thus, we have large absorption in both $x$ and $z$ directions. Also note that this behavior is completely different from QW detectors ${ }^{26,27}$ which allow light absorption in the $z$-direction $\left(\mathrm{A}_{\mathrm{z}}\right)$ only. In our case, all $A_{x}, A_{y}$, and $A_{z}$ components contribute to the detector responsivity. The parallel polarization shows this difference clearly. Diffracted light is still polarized in the $y$-direction, and thus, the light absorption is dominated by the $\mathrm{A}_{\mathrm{y}}$ component (Figs. 4(a)-4(d)) (but QW detectors cannot utilize this $A_{y}$ component). In our simulation, we used the anisotropic dielectric constants for the active region to account for the different absorption efficiencies of QDs into different directions.

Gradual tuning of the spectral response is more difficult for larger grating periods, but having more response peaks can help increase the overall detection performance. We elaborate on this point by evaluating the overall device performance (ODP). ODP is defined as the integrated detector responsivity over the whole spectrum. We investigate how the ODP changes with grating parameters $(p$ and $d)$. Figure 5(a) shows the calculated ODP as a function of $d / p$ for different grating periods $(p=1-4 \mu \mathrm{m})$ and incident polarizations. For a small period $(p=1 \mu \mathrm{m})$, the ODP does not show noticeable changes for both polarizations. But, for the larger periods $(p=2-4 \mu \mathrm{m})$, we can have significantly larger ODP enhancement (up to the ODP of $\sim 3.29$ and $\sim 4.24$ for parallel and perpendicular polarizations, respectively). The ODP of the unpatterned detector is 1.71 for a $1.5 \mu \mathrm{m}$ thick top contact $(d=1 \cdot p)$ and 1.84 for a $1 \mu \mathrm{m}$ thick top contact $(d=0 \cdot p)$. Compared to the unpatterned detector (the ODP of 1.71), the enhancement can be greater than $92 \%$ and $148 \%$ for parallel and perpendicular polarizations, respectively.

Figure 5(b) shows the ODP for unpolarized incident light (i.e., the average of two polarizations). Again, we clearly see the general trend: for a small periodicity $(p=1 \mu \mathrm{m})$, the ODP remains almost the same as that of the unpatterned device, but for a larger periodicity $(p=2-4 \mu \mathrm{m})$, the averaged ODP varies significantly depending on the ratio $d / p$, and a large enhancement can be achieved. This is also consistent in the overall sensitivity change in Fig. 2; we have more green regions representing enhanced sensitivity for the larger grating periods. We also represent the ODP data using a $2 \mathrm{D}$ colormap (Fig. 6). It clearly shows how the ODP changes with grating parameters for broad, unpolarized IR radiation (i.e., under a realistic measurement condition). The $p$ and $d / p$ values producing the same ODP are indicated as dotted contour lines. As the grating period $p$ increases, we have more response peaks, and the ODP increases (i.e., the color changes from blue to red). The ODP can reach beyond 3, depending on grating parameters. Although we only obtain a tiny ODP enhancement for small periods (around $p \sim 1 \mu \mathrm{m}$ ), it is still valuable for spectral response control, as discussed above.

Finally, we consider the effect of grating thickness. So far we have assumed that the grating thickness is fixed at $0.5 \mu \mathrm{m}$ (Fig. 1). But, when this thickness varies, the spectral responsivity is also altered due to changes in the FP resonance and diffraction strength. Figure 7 shows the simulated

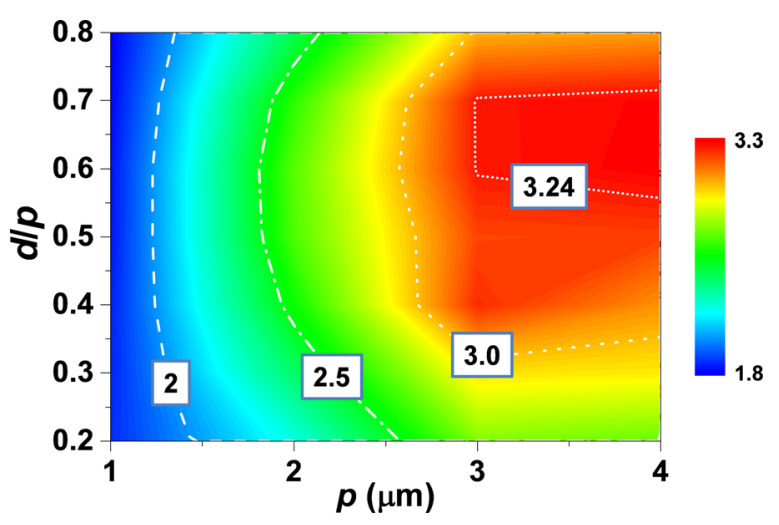

FIG. 6. A 2-dimensional color map of the ODP for unpolarized light as a function of the grating period $p$ and the ratio $d / p$. As the grating period $p$ increases, we have more response peaks and the ODP increases (i.e., the color changes from blue to red). The ODP can increase above 3 for a certain range of grating parameters. With small grating periods (around $p \sim 1 \mu \mathrm{m}$ ), we only obtain a tiny overall performance enhancement, but it is still useful for spectral response control. 


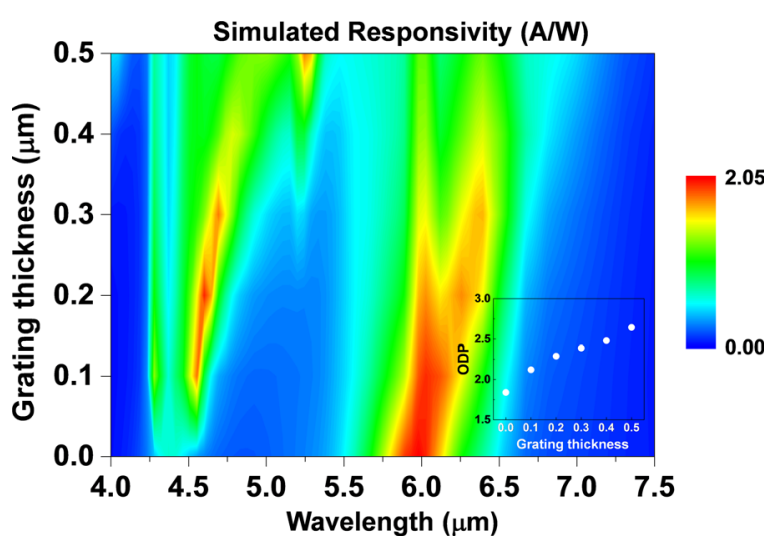

FIG. 7. Responsivity colormap for the grating period $p=2 \mu \mathrm{m}$ and pattern width $d=0.5 \cdot p$ for unpolarized incident light. It shows how spectral response changes when the grating thickness increases from 0 to $0.5 \mu \mathrm{m}$. The inset shows the ODP as a function of grating thickness. The ODP gradually increases for the given thickness range.

responsivity colormap for the grating period $p=2 \mu \mathrm{m}$ and width $d=0.5 \cdot p$ for unpolarized incident light. It shows how spectral response changes when the grating thickness increases from 0 to $0.5 \mu \mathrm{m}$. The inset in Fig. 7 shows the integrated responsivity (i.e., ODP) as a function of grating thickness. We find that the ODP gradually increases for the thickness range we considered.

In this work, we considered $1 \mathrm{D}$ gratings which can be easily patterned on a semiconductor contact layer. More elaborate $2 \mathrm{D}$ grating structures can be also designed and employed to increase the ODP further. However, our work clearly demonstrates that simple 1D structures provide significant control of spectral response and detection enhancement.

\section{CONCLUSION}

In conclusion, we have demonstrated that grating patterning on a semiconductor contact layer can be a simple and effective way to control the spectral responsivity with a minimal increase in device complexity. We also obtained enhancement of the overall detector performance for optimized grating parameters. This pixel-level grating structure is straightforward to pattern and compatible with current IR detector fabrication processes. We expect our design and analysis can lead to IR photodetectors useful for various IR imaging applications.

\section{ACKNOWLEDGMENTS}

S.K. acknowledges the support through Contract Nos. FA4600-06-0003 and FA9453-13-1-0284. Y.C.J. acknowledges the supports from Inha University Research Grant (No. INHA-47296), the National Research Foundation of Korea (NRF) Grant funded by the Korea government (Ministry of Science, ICT \& Future Planning) (No. 2008-0061893), and the MSIP (Ministry of Science, ICT\&Future Planning), Korea, under the ITRC (Information Technology Research Center) support Program (No. NIPA-2013-H0301-13-1010) supervised by the NIPA (National IT Industry Promotion Agency). S.J.L. acknowledges the support from Korea
Research Institute of Standards and Science grant, JP20120001.

${ }^{1}$ A. Rogalski, J. Antoszewski, and L. Faraone, J. Appl. Phys. 105, 091101 (2009).

${ }^{2}$ E. L. Dereniak and G. Boreman, Infrared Detectors and Systems (Wiley, 1996).

${ }^{3}$ P. R. Norton, J. B. Campbell, S. B. Horn, and D. A. Reago, Proc. SPIE 4130, 226-236 (2000)

${ }^{4}$ M. N. Abedin, T. F. Refaat, I. B. Bhat, Y. Xiao, S. V. Bandara, and S. D. Gunapala, Proc. SPIE 5543, 239-247 (2004).

${ }^{5}$ C.-C. Wang and S.-D. Lin, J. Appl. Phys. 113, 213108 (2013).

${ }^{6}$ I. M. Baker and R. A. Ballingall, Proc. SPIE 510, 121-129 (1985).

${ }^{7}$ T. E. Vandervelde, M. C. Lenz, E. Varley, A. Barve, J. Shao, R. Shenoi, D. A. Ramirez, W.-Y. Jang, Y. D. Sharma, and S. Krishna, IEEE J. Sel. Top. Quantum Electron. 14, 1150-1161 (2008).

${ }^{8}$ P. J. Miller and C. C. Hoyt, Proc. SPIE 2345, 354-365 (1995).

${ }^{9}$ C. M. Snively, S. Katzenberger, G. Oskarsdottir, and J. Lauterbach, Opt. Lett. 24, 1841-1843 (1999).

${ }^{10}$ S. Krishna, D. Forman, S. Annamalai, P. Dowd, P. Varangis, T. Tumolillo, A. Gray, J. Zilko, K. Sun, M. Liu, J. Campbell, and D. Carothers, Appl. Phys. Lett. 86, 193501 (2005).

${ }^{11}$ S. Krishna, D. Forman, S. Annamalai, P. Dowd, P. Varangis, T. Tumolillo, A. Gray, J. Zilko, K. Sun, M. Liu, J. Campbell, and D. Carothers, Phys. Status Solidi C 3, 439-443 (2006).

${ }^{12}$ G. Destefanis, J. Baylet, P. Ballet, P. Castelein, F. Rothan, O. Gravrand, J. Rothman, J. P. Chamonal, and A. Million, J. Electron. Mater. 36, 1031-1044 (2007).

${ }^{13}$ E. Varley, M. Lenz, S. J. Lee, J. S. Brown, D. A. Ramirez, A. Stintz, and S. Krishna, Appl. Phys. Lett. 91, 081120 (2007).

${ }^{14}$ Z. Yu, G. Veronis, S. Fan, and M. L. Brongersma, Appl. Phys. Lett. 89, 151116 (2006).

${ }^{15}$ J. Rosenberg, R. V. Shenoi, T. E. Vandervelde, S. Krishna, and O. Painter, Appl. Phys. Lett. 95, 161101 (2009).

${ }^{16}$ C.-C. Chang, Y. D. Sharma, Y.-S. Kim, J. A. Bur, R. V. Shenoi, S. Krishna, D. Huang, and S.-Y. Lin, Nano Lett. 10, 1704-1709 (2010).

${ }^{17}$ S. J. Lee, Z. Ku, A. Barve, J. Montoya, W.-Y. Jang, S. R. J. Brueck, M. Sundaram, A. Reisinger, S. Krishna, and S. K. Noh, Nat. Commun. 2, 286 (2011).

${ }^{18}$ Y. D. Sharma, Y. C. Jun, J. O. Kim, I. Brener, and S. Krishna, Opt. Commun. 312, 31 (2014).

${ }^{19}$ S. Hellström, Z.-H. Chen, Y. Fu, M. Qiu, R. Soltanmoradi, Q. Wang, and J. Y. Andersson, Appl. Phys. Lett. 96, 231110 (2010).

${ }^{20}$ M. S. Shishodia, P. V. V. Jayaweera, S. G. Matsik, A. G. U. Perera, H. C. Liu, and M. Buchanan, Photonic Nanostruct. 9, 95 (2011).

${ }^{21}$ J. Wang, X. Chen, Z. Li, and W. Lu, J. Opt. Soc. Am. B 27, 2428-2432 (2010).

${ }^{22}$ S. Krishna, J. Phys. D 38, 2142-2150 (2005).

${ }^{23}$ G. T. Liu, A. Stintz, H. Li, T. C. Newell, A. L. Gray, P. M. Varangis, K. J. Malloy, and L. F. Lester, IEEE J. Quantum Electron. 36, 1272-1279 (2000).

${ }^{24}$ S. Raghavan, P. Rotella, A. Stintz, B. Fuchs, S. Krishna, C. Morath, D. A. Cardimona, and S. W. Kennerly, Appl. Phys. Lett. 81, 1369-1371 (2002).

${ }^{25}$ A. V. Barve, S. J. Lee, S. K. Noh, and S. Krishna, Laser Photonics Rev. 4, 738-750 (2010).

${ }^{26}$ J. Y. Andersson, L. Lundqvist, and Z. F. Paska, Appl. Phys. Lett. 58, 2264-2266 (1991).

${ }^{27}$ L. Lundqvist, J. Y. Andersson, Z. F. Paska, J. Borglind, and D. Haga, Appl. Phys. Lett. 63, 3361-3363 (1993).

${ }^{28}$ J. O. Kim, S. Sengupta, A. V. Barve, Y. D. Sharma, S. Adhikary, S. J. Lee, S. K. Noh, M. S. Allen, J. W. Allen, S. Chakrabarti, and S. Krishna, Appl. Phys. Lett. 102, 011131 (2013).

${ }^{29}$ Z. Ku, W.-Y. Jang, J. Zhou, J. O. Kim, A. V. Barve, S. Silva, S. Krishna, S. R. J. Brueck, R. Nelson, A. Urbas, S. Kang, and S. J. Lee, Opt. Express 21, 4709-4716 (2013).

${ }^{30}$ See www.cst.com for CST Microwave Studio.

${ }^{31}$ J. D. Jackson, Classical Electrodynamics, 3rd ed. (Wiley, New York, 1999).

${ }^{32}$ W. G. Spitzer and J. M. Whelan, Phys. Rev. 114, 59-63 (1959).

${ }^{33}$ S. Law, D. C. Adams, A. M. Taylor, and D. Wasserman, Opt. Express 20, 12155-12165 (2012).

${ }^{34}$ W. Liu, Y. Li, H. Jiang, Z. Lai, and H. Chen, Opt. Lett. 38, 163-165 (2013).

${ }^{35}$ G. Bao and K. Huang, J. Opt. Soc. Am. A 22, 1408-1413 (2005).

${ }^{36}$ W. Liu, Z. Lai, H. Guo, and Y. Liu, Opt. Lett. 35, 865-867 (2010). 